\title{
Clinical Significances of Preoperative Classification of Intrahepatic Cholangiocarcinoma: Different Characteristics of Perihilar vs. Peripheral ICC
}

\author{
YO-ICHI YAMASHITA ${ }^{1,2}$, HUANLIN WANG $^{3}$, TAKESHI KURIHARA ${ }^{3}$, EIJI TSUJITA ${ }^{2}$, AKIHIRO NISHIE ${ }^{4}$, \\ KATSUNORI IMAI ${ }^{1}$, DAISUKE HASHIMOTO ${ }^{1}$, AKIRA CHIKAMOTO ${ }^{1}$, SHINICHI AISHIMA ${ }^{5}$ and HIDEO BABA ${ }^{1}$ \\ ${ }^{1}$ Department of Gastroenterological Surgery, Kumamoto University, Kumamoto, Japan; \\ ${ }^{2}$ Department of Hepato-Biliary-Pancreatic Surgery, National Kyushu Cancer Center, Fukuoka, Japan; \\ ${ }^{3}$ Department of Surgery and Science, Kyushu University, Fukuoka, Japan; \\ ${ }^{4}$ Department of Clinical Radiology, Kyushu University, Fukuoka, Japan; \\ ${ }^{5}$ Department of Pathology and Microbiology, Saga University, Saga, Japan
}

\begin{abstract}
Background: The aim of this study was to evaluate the clinical significance of preoperative classification of intrahepatic cholangiocarcinoma (ICC) into perihilar and peripheral types using dynamic computed tomography $(C T)$. Patients and Methods: A retrospective cohort study was performed to analyze the differences in clinical characteristics between perihilar and peripheral ICC samples from patients between 1990-2014. Results: A total of 87 patients were divided into three ICC subtypes; perihilar $(n=34)$, peripheral $(n=44)$, and unclassifiable ICC $(n=9)$. The positive rates of pathological lymphatic infiltration (ly) $(p=0.02)$ and perineural invasion $(p n)(p<0.0001)$ were significantly higher in perihilar ICC. There was no significant difference in the disease-free survival rate $(p=0.2268)$; however, the overall survival rate of perihilar ICC was significantly worse $(p=0.0031)$. The rate of systemic recurrence ( $>3$ nodules) was significantly higher in perihilar ICC ( $p=0.0135)$. Conclusion: In perihilar ICC, the local tumor invasions such as ly and pn were more frequent. Therefore, it is important in such cases to attempt to achieve a sufficient tumor margin. Systemic recurrences were more frequent in perihilar ICC, so perioperative chemotherapy should be conducted as well.
\end{abstract}

This article is freely accessible online.

Correspondence to: Yo-ichi Yamashita, MD, Ph.D., F.A.C.S., Department of Gastroenterological Surgery, Graduate School of Life Science, Kumamoto University, 1-1-1, Honjyo, Chuo-ku, Kumamoto 860-8556, Japan. Tel: +81 963735211, Fax: +81 963714378, e-mail: y-yama@kumamoto-u.ac.jp

Key Words: Intrahepatic cholangiocarcinoma, perihilar type, peripheral type.
Even though intrahepatic cholangiocarcinoma (ICC) is a relatively rare disease, it is the second most common primary liver malignant tumor after hepatocellular carcinoma (HCC), and has a relatively highly prevalence in Asia and the U.S.A., with over $1 / 100,000$ population ratio (1). In addition, the incidence of ICC has rapidly increased by $165 \%$ in the last 30 years in the U.S.A., and surgical resection remains the only treatment available with curative intent (2). However, surgical results for ICC remain unsatisfactory with a five-year survival rate of $58 \%$, even in $\mathrm{R} 0$ resected cases (3). Lymph node metastasis has been reported to be the most significant poor prognostic factor for ICC, and extended lymphadenectomy does not have a survival impact (3-5). While lymphatic invasion is the major mode of cancer spreading for ICC (6), intrahepatic metastasis caused by venous spread is another important mode of spreading $(4,5)$.

ICCs are composed of heterogenous carcinomas arising from different anatomical liver sites. ICC arises from the epithelial cells of the biliary tree, from either intrahepatic large bile ducts or smaller bile ducts, such as the septal and interlobular ducts. Ebata et al. clearly defined as perihilar cholangiocarcinoma, a tumor involving the hilar bile duct with a liver mass (7). We proposed the classification of ICC based on the pathological findings in perihilar large duct type and peripheral small duct type subtypes $(8,9)$. Perihilar ICC arises from the peribiliary gland, and peripheral ICC from the canals of Hering (9); the two subtypes have different molecular and clinicopathological characteristics and patient prognoses $(8,9)$. However, pathological classification currently contributes only whether or not to plan adjuvant chemotherapy. Therefore, it is important to construct a preoperative classification that can be used to modify surgical plans and so on to improve patients' prognosis for ICC. 
In this study, we tried to classify ICC preoperatively into two subtypes, perihilar and peripheral, using dynamic computed tomography (CT). The accuracy of this classification was evaluated by comparison with pathological findings. The differences in clinical characteristics of the two subtypes, such as mode of cancer spreading and recurrence patterns, were analyzed to improve treatment strategy for ICC.

\section{Patients and Methods}

Patients. A total of 87 hepatic resections for mass forming dominant ICC, confirmed by pathological diagnosis and performed at the Department of Surgery and Sciences, Kyushu University Hospital, between January 1990 and March 2014. The intraductal growth type of ICC, without invasion to liver parenchyma, was excluded from this study. The medical records of patients in this series were followed until March 2015, with a median follow-up period of 69 months.

Preoperative classification using dynamic CT. All patients underwent dynamic CT preoperatively. Because the retrospective nature of this investigation dated back to 1990, a variety of CT scanners and contrast methods were used in the series (10). According to preoperative dynamic CT findings, we tried to classify ICC into one of the two subtypes; perihilar or peripheral. Our criteria of this classification were as follows; 1) Tumor's main location. If the main tumor was judged to occur at the second or third branches of an intrahepatic bile duct, the case was classified as a perihilar type; in contrast, if the main tumor was judged to be at peripheral liver, the case was considered peripheral. 2) Intrahepatic bile duct dilatation. If the case was accompanied by intrahepatic bile duct dilatation, it was classified as the perihilar type.

Surgical techniques and follow-up methods. The details of our surgical techniques and patient follow-up methods have been reported previously $(3,4,6)$. Major hepatectomy $(\mathrm{Hx})$ with bile duct resection was performed when bile duct invasion of ICC was suspected to affect the first hepatic duct. Partial Hx was performed in case of peripheral ICC without bile duct invasion. If we considered it would be better to confirm the surgical margins, we presented the resected stump for frozen pathology (3). The right and left lobes of the liver have different routes of lymphatic drainage; therefore, the style of lymph node dissection was different according to the tumor location on the right or left lobe (4). Five patients $(5.7 \%)$ were diagnosed as HCC preoperatively, and underwent only partial $\mathrm{Hx}$.

Statistical analysis. Continuous variables were expressed as means \pm S.D. and compared using a Student's $t$-test. Categorical variables were compared using either the $\chi^{2}$ test or the Fisher's exact test, as appropriate. Any death that occurred in the hospital after $\mathrm{Hx}$ was recorded as a mortality. Complications were evaluated with the Clavien's classification, and those with a score of Grade II or more were defined as positive. The OS and DFS curves were generated by the Kaplan-Meier method and compared by the log-rank test. All analyses were performed with JMP ${ }^{\circledR}$ Pro 9.0.2 (SAS Institute Inc., Cary, NC, USA). $p$-Values of less than 0.05 were considered to indicate statistical significance.

\section{Results}

Preoperative classification of ICC using dynamic CT. We could classify 78 ICC patients $(90 \%)$ as follows; perihilar ICC in 34 patients $(39 \%)$ and peripheral ICC in 44 patients (51\%). Nine patients $(10 \%)$ could not be classified as either perihilar or peripheral ICC (unclassifiable type), because the tumor location of the ICC extended from perihilar to peripheral sites without bile duct dilatation. Typical dynamic CT findings for perihilar, peripheral, and unclassified types are shown in Figures 1, 2, and 3, respectively.

Comparisons of classification by preoperative dynamic CT and postoperative pathological evaluations are summarized in Table I. All nine patients in the unclassifiable type were pathologically perihilar ICC. The accuracy of our preoperative classification was a relatively high $86 \%$.

Comparison of patient background characteristics. The results of a comparison of patient background characteristics between the two subtypes are summarized in Table II. The positive rate of hepatitis $\mathrm{B}$ virus surface antigen (HBs-Ag) was significantly higher in peripheral ICC (perihilar, 3\%; peripheral, 23\%; $p=0.0129)$. There were also significant differences in total bilirubin (T-bil) (perihilar, $1.8 \pm 2.6 \mathrm{mg} / \mathrm{dl}$; peripheral, $0.7 \pm 0.3 \mathrm{mg} / \mathrm{dl} ; \quad p=0.0088)$ and asparate aminotransferase (ALT) levels (perihilar, 80.9 $\pm 128.4 \mathrm{mg} / \mathrm{dl}$; peripheral, $31.9 \pm 25.0 \mathrm{mg} / \mathrm{dl} ; p=0.0155)$.

Comparison of short-term surgical outcomes. The results of a comparison of short-term surgical outcomes are summarized in Table III. Operation time was significantly prolonged in perihilar ICC (perihilar, 521 $\pm 158 \mathrm{~min}$; peripheral, 343 $\pm 1,139 \mathrm{~min}$; $p<0.0001$ ), and intra-operative blood loss was significantly larger (perihilar, 2413 $\pm 358 \mathrm{~g}$; peripheral, 890 $\pm 305 \mathrm{~g} ; p=0.0018$ ). Therefore, the intra-operative transfusion rate in perihilar ICC was significantly higher (perihilar, 47\%; peripheral, 23\%; $p=0.0086$ ). Major $\mathrm{Hx}$ (perihilar, 100\%; peripheral, 41\%; $p<0.0001$ ), biliary resection and reconstruction (perihilar, 59\%; peripheral, $9 \% ; p<0.0001$ ) and lymph node dissection (perihilar, $76 \%$; peripheral, $41 \%$; $p=0.0014$ ) were performed significantly more in perihilar ICC. However, the positive rate of surgical margin was significantly higher in perihilar ICC irrespective of these extended resections (perihilar, 24\%; peripheral, 2\%; $p=0.0025)$.

Both mortality (perihilar, $9 \%$; peripheral, $0 \% ; p=0.0234$ ) and morbidity rate (perihilar, $62 \%$; peripheral, $25 \% ; p=0.0010$ ) were significantly higher in perihilar ICC, and the mean duration of hospital stay was significantly prolonged in this group (perihilar, $34 \pm 22$ days; peripheral, $22 \pm 21$ days; $p=0.0176$ ).

Comparisons of tumor-related factors. Results from the comparison of tumor-related factors are summarized in Table IV. The positive rate of poorly differentiated ICC was 
A

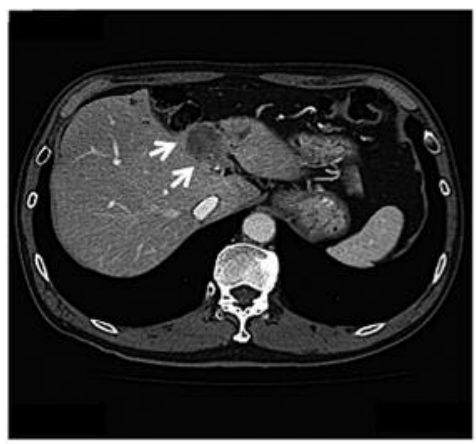

B

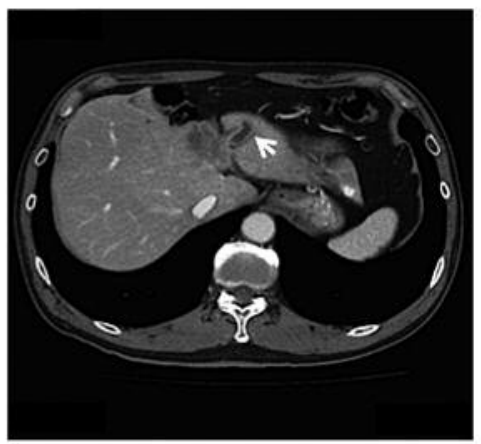

Figure 1. Typical dynamic CT findings of perihilar ICC (portal phases). (A) Arrows indicate the main tumor close to the umbilical portion, (B) arrow indicates the dilatation of the intrahepatic bile duct.
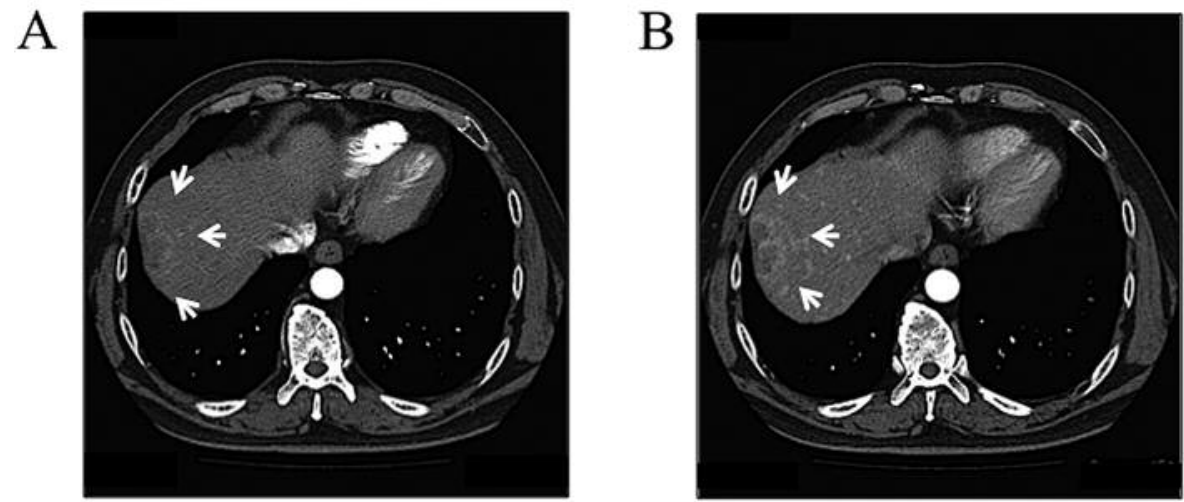

Figure 2. Typical dynamic CT findings of peripheral ICC. Arrows indicate the main tumor in the arterial phase (A) and in the late arterial phase (B).
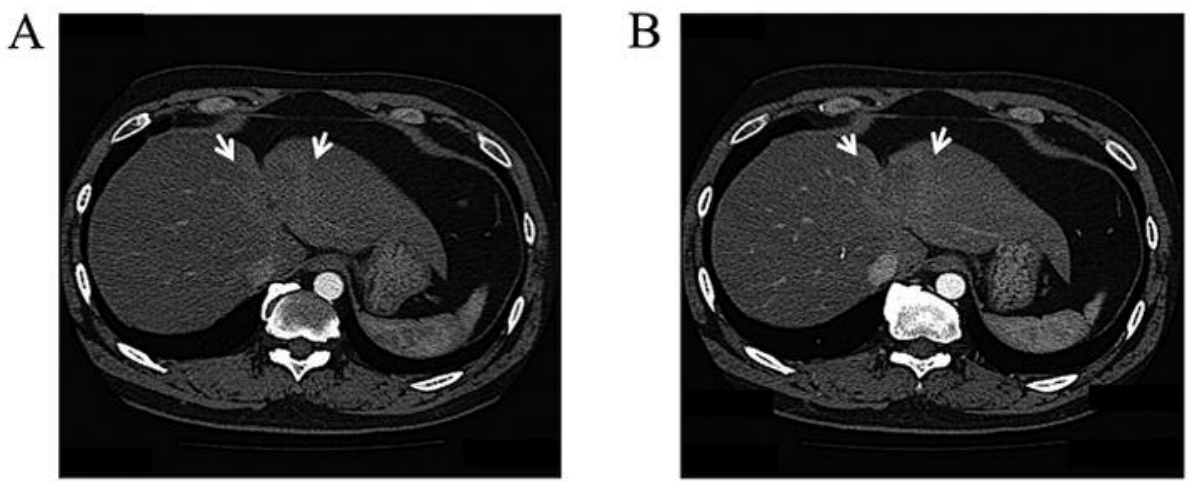

Figure 3. Typical dynamic CT findings of unclassifiable ICC (A; arterial phase, B; portal phase). Arrows indicate the main tumor, which extended from the umbilical portion (A) to the peripheral $(B)$.

significantly higher in peripheral ICC (perihilar, 44\%; peripheral, $61 \% ; p=0.0253$ ), Meanwhile, positive rates of pathological lymphatic infiltration (ly) (perihilar, 50\%; peripheral, 25\%; $p=0.02$ ) and perineural invasion (pn) (perihilar, 85\%; peripheral, 30\%; $p<0.0001$ ) were significantly higher in perihilar ICC. There were no significant differences in the positive rate of pathological lymph node metastasis (perihilar, 38\%; peripheral, 25\%; $p=0.2102$ ). The value of carbohydrate antigen 19-9 (CA19-9) was significantly higher in perihilar ICC (perihilar, 2,360 $\pm 1,183 \mathrm{U} / \mathrm{ml}$; peripheral, 
Table I. The acccuracy of preoperative classification of perihilar type vs. peripheral-type ICC using dynamic CT.

\begin{tabular}{lrccc}
\hline $\begin{array}{l}\text { Pathological } \\
\text { classification }\end{array}$ & \multicolumn{4}{c}{ CT classification } \\
\cline { 2 - 5 } & Perihilar & Peripheral & Unclassifiable & Total \\
\hline Perihilar & 32 & 1 & 9 & 42 \\
Peripheral & 2 & 43 & 0 & 45 \\
Total & 34 & 44 & 9 & 87 \\
\hline
\end{tabular}

Accuracy: $[78 / 87 \times(32+43 / 78)] \times 100=86 \%$

Table II. Comparison of patient background characteristics.

\begin{tabular}{lccc}
\hline Variables & $\begin{array}{c}\text { Pehihilar } \\
(\mathrm{n}=34)\end{array}$ & $\begin{array}{c}\text { Peripheral } \\
(\mathrm{n}=44)\end{array}$ & $p$-Value \\
\hline Age & $62.8 \pm 8.6$ & $61.1 \pm 11.7$ & 0.4836 \\
Male/Female & $30 / 14$ & $23 / 11$ & 0.9600 \\
BMI & $21.5 \pm 3.0$ & $22.2 \pm 3.8$ & 0.3827 \\
DM (+) (\%) & $4(12 \%)$ & $11(25 \%)$ & 0.1440 \\
HBs-Ag (+) (\%) & $1(3 \%)$ & $10(23 \%)$ & 0.0129 \\
HCV-Ab (+) (\%) & $4(12 \%)$ & $8(18 \%)$ & 0.7273 \\
T-bil (mg/dl) & $1.8 \pm 2.6$ & $0.7 \pm 0.3$ & 0.0088 \\
Alb (g/dL) & $4.1 \pm 0.4$ & $4.0 \pm 0.4$ & 0.4680 \\
AST (IU/L) & $44.8 \pm 24.2$ & $34.5 \pm 22.5$ & 0.0562 \\
ALT (IU/L) & $80.9 \pm 128.4$ & $31.9 \pm 25.0$ & 0.0155 \\
PT (\%) & $93.9 \pm 12.2$ & $90.0 \pm 15.3$ & 0.2140 \\
Plt $(\times 104 / \mu l)$ & $12.4 \pm 12.4$ & $7.4 \pm 10.0$ & 0.0553 \\
ICGR-15 (\%) & $10.3 \pm 5.6$ & $12.8 \pm 7.6$ & 0.1102 \\
Child A (\%) & $27(79 \%)$ & $41(93 \%)$ & 0.0709 \\
\hline
\end{tabular}

BMI; Body mass index, DM; diabetes mellitus, HBs-Ag; hepatitis B virus surface antigen, $\mathrm{HCV}-\mathrm{Ab}$; hepatitis $\mathrm{C}$ antibody, T-bil; total bilirubin, Alb; albumin, AST; aspartate aminotransferase, ALT; alanine aminotransferase, PT; prothrombin time, Plt; platelet count, ICGR-15; indocyanin green retention rate at $15 \mathrm{~min}$.

$408 \pm 1,621 \mathrm{U} / \mathrm{ml} ; p=0.0487)$. There were no significant differences in the positive rate of pathological portal venous and/or hepatic venous infiltration (vp/vv), or pathological intrahepatic metastasis (im). The positive rate of histological cirrhosis (lc) in peripheral ICC was significantly higher (perihilar, 0\%; peripheral, 27\%, $p<0.0001$ ).

Comparisons of DFS and OS rates. The DFS and OS curves after operation in the two subtypes are illustrated in Figure 4. There was no significant difference in DFS rate $(p=0.2268)$; the two-year DFS rate of perihilar ICC was $32 \%$, and that of peripheral ICC was $46 \%$. The OS rate in perihilar ICC was significantly worse $(p=0.0031)$, and the five-year OS rate in peripheral ICC reached $51 \%$ compared to perihilar ICC in which remained low at $27 \%$.
Table III. Comparison of short-term surgical outcomes.

\begin{tabular}{lccc}
\hline Variables & $\begin{array}{c}\text { Perihilar } \\
(\mathrm{n}=34)\end{array}$ & $\begin{array}{c}\text { Peripheral } \\
(\mathrm{n}=44)\end{array}$ & $p$-Value \\
\hline Surgical outcomes & & & \\
$\quad$ Operation time (min) & $521 \pm 158$ & $343 \pm 1139$ & $<0.0001$ \\
Blood loss (g) & $2413 \pm 358$ & $890 \pm 305$ & 0.0018 \\
Trasfusion (+) (\%) & $16(47 \%)$ & $10(23 \%)$ & 0.0086 \\
Major Hx (\%) & $34(100 \%)$ & $18(41 \%)$ & $<0.0001$ \\
Lymph node dissection (+) (\%) & $26(76 \%)$ & $18(41 \%)$ & 0.0014 \\
Biliary reconstruction (+) (\%) & $20(59 \%)$ & $4(9 \%)$ & $<0.0001$ \\
R1/2(\%) & $9(26 \%)$ & $8(18 \%)$ & 0.3809 \\
sm+ (\%) & $8(24 \%)$ & $1(2 \%)$ & 0.0025 \\
Post-operative courses & & & \\
Mortality (\%) & $3(9 \%)$ & $0(0 \%)$ & 0.0234 \\
Morbidity (\%) & $21(62 \%)$ & $11(25 \%)$ & 0.0010 \\
Hospital stay (days) & $34 \pm 22$ & $22 \pm 21$ & 0.0176 \\
\hline
\end{tabular}

Hx; Hepatectomy, sm; surgical margin.

Table IV. Comparison of tumor-related factors.

\begin{tabular}{lccc}
\hline Variables & $\begin{array}{c}\text { Perihilar } \\
(\mathrm{n}=34)\end{array}$ & $\begin{array}{c}\text { Peripheral } \\
(\mathrm{n}=44)\end{array}$ & $p$-Value \\
\hline Tumor diameter $(\mathrm{cm})$ & $4.0 \pm 1.8$ & $4.5 \pm 2.1$ & 0.2313 \\
Solitary/multiple & $24 / 10$ & $30 / 14$ & 0.8194 \\
n (+) (\%) & $13(38 \%)$ & $11(25 \%)$ & 0.2102 \\
Stage III or IVA (\%) & $26(76 \%)$ & $37(84 \%)$ & 0.3989 \\
Poorly dif. (\%) & $15(44 \%)$ & $27(61 \%)$ & 0.0253 \\
ly $(+)(\%)$ & $17(50 \%)$ & $11(25 \%)$ & 0.0200 \\
pn $(+)(\%)$ & $29(85 \%)$ & $13(30 \%)$ & $<0.0001$ \\
s (+) $(\%)$ & $19(56 \%)$ & $32(73 \%)$ & 0.1214 \\
vp/vv $(+)(\%)$ & $21(62 \%)$ & $23(68 \%)$ & 0.4010 \\
im (+) (\%) & $18(53 \%)$ & $14(32 \%)$ & 0.1102 \\
b (+) (\%) & $14(41 \%)$ & $12(27 \%)$ & 0.2440 \\
CEA (ng/ml) & $7.8 \pm 20.4$ & $3.5 \pm 5.1$ & 0.1870 \\
CA19-9 (U/ml) & $2360 \pm 1183$ & $408 \pm 1621$ & 0.0483 \\
lc $(+)(\%)$ & $0(0 \%)$ & $12(27 \%)$ & $<0.0001$ \\
\hline
\end{tabular}

n; Pathological lymph node metastasis, poorly dif.; poorly differentiated, ly; pathological lymphatic infiltration, pn; pathological perinural invasion, s; pathological serosal invasion, vp; pathological portal venous infiltration, vv; pathological venous infiltration, im; pathological intrahepatic metastasis, b; pathological bile duct invasion, CEA; carcinoembryonic antigen, CA19-9; carbohydrate antigen 19-9, lc; histological cirrhosis.

As for patients after R0 resection, Figure 5 shows the DFS and OS curves after operation in the two subtypes. Again, there was no significant difference in the DFS rate $(p=0.3898)$; the two-year DFS rate in perihilar ICC was $49 \%$, and that in peripheral ICC was $57 \%$. The OS rate in perihilar ICC was significantly worse $(p=0.0033)$, and the five-year OS rate of peripheral ICC reached 63\%; however that in perihilar ICC remained low at 39\%. 
A

$\underline{\text { DFS }}$

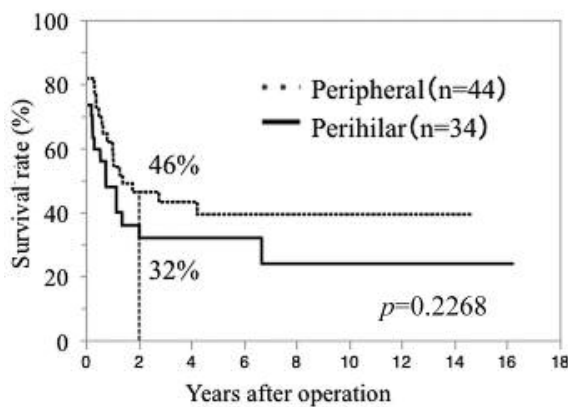

B

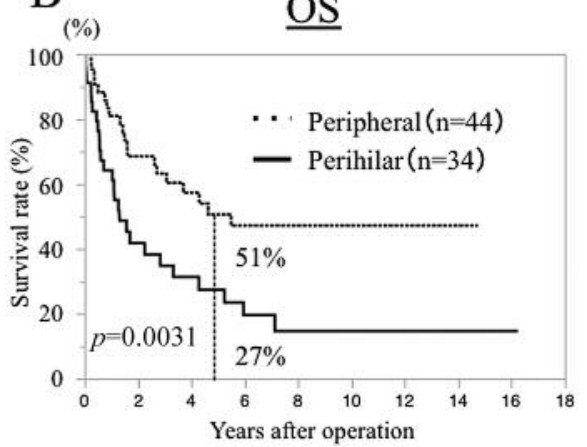

Figure 4. The DFS and OS curves of the two subtypes in all patients after operation. There was no significant difference in the DFS rate (p=0.2268) (A). The OS rate in perihilar ICC was significantly worse $(p=0.0031)$ and the five-year OS rate of peripheral ICC reached $51 \%$; however, in perihilar ICC remained low at $27 \%$ (B).

A

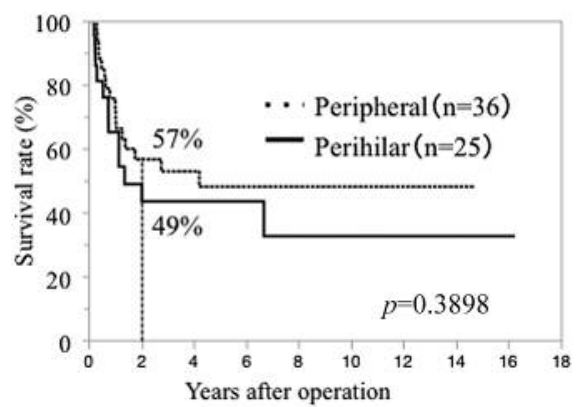

B $\underline{\text { OS }}$

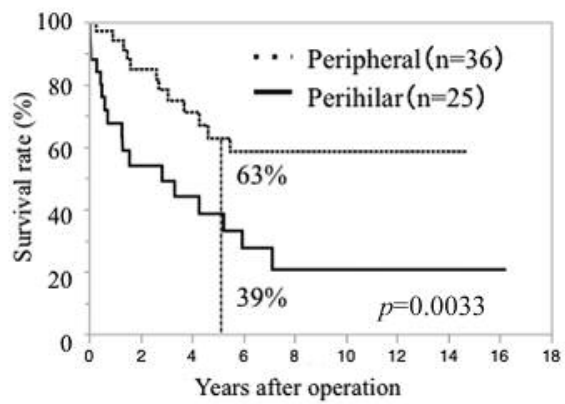

Figure 5. The DFS and OS curves of the two subtypes in patients after R0 operation. There was no significant difference in the DFS rate (p=0.3898) (A). The OS rate in perihilar ICC was significantly worse ( $p=0.0033)$ and the five-year OS rate of peripheral ICC reached $63 \%$; however, that in perihilar ICC remained low at 39\% (B).

Comparisons between the two subtypes in the recurrence pattern after R0 resection are summarized in Table V. The recurrence rate was similar in both; $35 \%$ (12 patients) in perihilar ICC, and 36\% (16 patients) in peripheral ICC. Among patients with a recurrence, when the recurrence was divided into a pattern such as localized recurrence $(\leq 3$ nodules) and systemic recurrence ( $>3$ nodules), the rate of systemic recurrence in perihilar ICC was significantly higher (perihilar, 92\%; peripheral, 50\%; $p=0.0135$ ). Lymph noderelated recurrence in perihilar ICC was as high as 50\% $(6$ patients). On the other hand, liver-related recurrence in peripheral ICC was as high as $75 \%$ (12 patients).

\section{Discussion}

There have been several reports including our own concerning the classification of ICC into hilar and peripheral ICC according to the location of the main tumor $(8,9,11$ 15). However, this is the first report to investigate the
Table V. Comparison of recurrence pattern after $R O$ resection.

\begin{tabular}{lcc}
\hline Recurrence pattern & $\begin{array}{c}\text { Perihilar } \\
(\mathrm{n}=34)\end{array}$ & $\begin{array}{c}\text { Peripheral } \\
(\mathrm{n}=44)\end{array}$ \\
\hline Recurrence $(+)$ & $12(35 \%)$ & $16(36 \%)$ \\
Localized recurrence $(+)(\leq 3$ nodules) & $1(8 \%)$ & $8(50 \%)$ \\
Liver & 0 & 4 \\
Lung & 0 & 1 \\
Liver and Lung & 1 & 3 \\
Systemic recurrence $(+)(>3$ nodules) & $11(92 \%)$ & $8(50 \%)$ \\
Liver & 2 & 5 \\
Lung & 0 & 1 \\
Lymph nodes & 6 & 0 \\
Peritoneal dissemination & 3 & 2 \\
\hline
\end{tabular}

clinical significance of preoperative classification of ICC. Compared to the pathological classification $(8,9)$, the accuracy of our simple preoperative classification of ICC 
using preoperative dynamic CT was relatively high $86 \%$. The tumor size of all nine unclassifiable ICCs was over $5 \mathrm{~cm}$, and all were classified as perihilar ICC by pathological evaluation. If we had automatically included unclassifiable ICC into the perihilar group, the accuracy of our preoperative classification would have increased to $97 \%$.

We previously reported that the two ICCs subtypes have different cellular origins; perihilar ICC's origin is from the peribiliary gland while peripheral ICC from the canals of Hering (9). Biliary chronic inflammation is considered one of the main causes of cancer development in perihilar ICC and viral hepatitis or cirrhosis in peripheral ICC $(8,9)$. In this report, the positive rate of $\mathrm{HBs}-\mathrm{Ag}(3 \%$ vs. $23 \%$; $p=0.0129)$ and lc (0 vs. 27\%; $p<0.0001)$ were significantly higher in peripheral ICC. Two recent papers suggest that ICC may arise directly from transdifferentiation of hepatocyte $(16,17)$. Therefore, peripheral ICC may develop from small bile ducts or hepatocytes. The relationship between HBV infection (odds ratio, OR; 2.66), cirrhosis (OR; 22.9) and ICC carcinogenesis were supported by two meta-analyses $(18,19)$. In addition, some studies have reported conflicting findings as to whether $\mathrm{HBV}, \mathrm{HCV}$ or both were associated with $\operatorname{ICC}(18,20)$. In this study, the significant increase of T-bil and ALT in perihilar ICC was likely caused not by liver dysfunction or hepatocytes inflammation but by obstructive liver injury, together with other variables such as the indocyanin green retention rate at $15 \mathrm{~min}$.

Due to tumor location, major Hx was significantly more frequently performed in perihilar ICC (41\% vs. $100 \%$; $p<0.0001)$, and the positive rate of performance in lymph node dissections ( $41 \%$ vs. $76 \% ; p=0.0014)$ and biliary reconstruction (9\% vs. 59\%; $p<0.0001)$ were also significantly higher in perihilar ICC. Despite these extended resections, the positive rate of surgical margin in perihilar ICC reached $24 \%$, and this rate was significantly higher than that in peripheral ICC $(2 \% ; p=0.0025)$. This might have been due to the more aggressive local tumor invasions in perihilar ICC such as ly $(50 \%$ vs. $25 \% ; p=0.0200)$ and pn $(80 \%$ vs. $30 \% ; p<0.0001)$. We should pay close attention to achieving wide tumor margins in both liver parenchyma and in bile duct in resections in perihilar ICC.

In all patients and in those with $\mathrm{R} 0$ resection, the $\mathrm{OS}$ rate is significantly better in peripheral ICC ( $p=0.0031, p=0.0033$, respectively); however there was no significant difference in DFS ( $p=0.2268, p=0.3898$, respectively). This discrepancy was likely caused by the difference in recurrence pattern. The major recurrence pattern of perihilar ICC was systemic ( $>3$ nodules; 92\%), and it was mainly involving lymph nodes $(50 \%)$. The mean value of CA19-9, which is reported to be the most important prognostic factor in ICC (5), was significantly higher in perihilar ICC than in peripheral ICC; therefore, perihilar ICC would have more potential to systemic spreading. On the other hand, the recurrence pattern of peripheral ICC was localized ( $\leq 3$ nodules; $50 \%$ ) and was mainly intrahepatic $(75 \%)$. Six patients $(38 \%)$ underwent surgical resection against recurrent tumors with curative intent, and this aggressive surgery for recurrence would lead to the better OS rate in peripheral $\operatorname{ICC}(21,22)$.

To improve patient survival in perihilar ICC, the addition of perioperative chemotherapy should be an option. However, there are contradictive results concerning the survival impacts of adjuvant chemotherapy for ICC $(23,24)$. Considering the high invasiveness of patients receiving major Hx for perihilar ICC, the neoadjuvant setting would be a preferable option for better patient survival after operation. On the other hand, recurrence in peripheral ICC is almost always intrahepatic. Therefore, perioperative chemotherapy via the hepatic artery is also an option towards improving survival (25). In addition, considering the recurrence pattern and the relatively low rate of ly and pn in peripheral ICC, liver transplantation with perioperative chemotherapy could be a feasible choice $(26,27)$.

According to the findings of dynamic CT and clinical futures, peripheral ICC's characteristics are similar to HCC. Actually, in our own cases, five patients (5.7\%) were preoperatively diagnosed as HCC. However, Table IV clearly shows that there are many different pathological findings such as the positive rate of ly $(25 \%)$ and pn $(30 \%)$, that are rarely seen in HCC. In addition, 11 cases $(25 \%)$ in peripheral ICC had lymph node metastasis. All tumor sizes of peripheral ICC with lymph node metastasis were over $5 \mathrm{~cm}$, and six cases $(55 \%)$ had multiple tumors. Probably, some peripheral ICCs have clinical characteristics similar to HCC; however, we want to emphasize the clear differences between peripheral ICC and HCC demonstrated in this study. Therefore, we should be careful in applying radiofrequency ablation in peripheral ICC (28).

In conclusion, we found that preoperative classification of ICC into perihilar and peripheral subtypes had a relatively high accuracy rate of $86 \%$. In perihilar ICC, the local tumor invasions such as ly and pn are more potent and therefore we should seek sufficient tumor margins against perihilar ICC. Systemic recurrence is also more frequent in perihilar ICC, so perioperative chemotherapy should be conducted to improve patients' prognosis.

\section{References}

1 Bridgewater J, Galle PR, Khan SA, Llovet JM, Park JW, Patel T, Pawlik TM and Gores GJ: Guidelines for the diagnosis and management of intrahepatic cholangiocarcinoma. J Hepatol 60: 1268-1289, 2014.

2 Aljiffry M, Abdulelah A, Walsh M, Peltekian K, Alwayn I and Molinari M: Evidence-based approach to cholangiocarcinoma: a systematic review of the current literature. J Am Coll Surg 208: 134-147, 2009. 
3 Yamashita Y, Taketomi A, Morita K, Fukuhara T, Ueda S, Sanefuji K, Iguchi T, Kayashima H, Sugimachi K and Maehara Y: The impact of surgical treatment and poor prognostic factors for patients with intrahepatic cholangiocarcinoma: retrospective analysis of 60 patients. Anticancer Res 28: 2353-2359, 2008.

4 Shimada M, Yamashita Y, Aishima S, Shirabe K, Takenaka K and Sugimachi K: Value of lymph node dissection during resection of intrahepatic cholangiocarcinoma. Br J Surg 88: 1463-1466, 2001.

5 Uchiyama K, Yamamoto M, Yamaue H, Ariizumi S, Aoki T, Kokudo N, Ebata T, Nagino M, Ohtsuka M, Miyazaki M, Tanaka E, Kondo S, Uenishi T, Kubo S, Yoshida H, Unno M, Imura S, Shimada M, Ueno M and Takada T: Impact of nodal involvement on surgical outcomes of intrahepatic cholangiocarcinoma: a multicenter analysis by the Study Group for Hepatic Surgery of the Japanese Society of Hepato-Biliary-Pancreatic Surgery. J Hepatobiliary Pancreat Sci 18: 443-452, 2011.

6 Shirabe K, Mano Y, Taketomi A, Soejima Y, Uchiyama H, Aishima S, Kayashima $H$, Ninomiya $M$ and Maehara $Y$ : Clinicopathological prognostic factors after hepatectomy for patients with mass-forming type intrahepatic cholangiocarcinoma: relevance of the lymphatic invasion index. Ann Surg Oncol 17: 1816-1822, 2010.

7 Ebata $T$, Kosuge $T$, Hirano S, Unno $M$, Yamamoto $M$, Miyazaki M, Kokudo N, Miyagawa S, Takada T and Nagino M: Proposal to modify the International Union Against Cancer staging system for perihilar cholangiocarcinomas. Br J Surg 101: 79-88, 2014.

8 Aishima S, Kuroda Y, Nishihara Y, Iguchi T, Taguchi K, Taketomi A, Maehara $\mathrm{Y}$ and Tsuneyoshi M: Proposal of progression model for intrahepatic cholangiocarcinoma: clinicopathologic differences between hilar type and peripheral type. Am J Surg Pathol 31: 1059-1067, 2007.

9 Aishima S and Oda Y: Pathogenesis and classification of intrahepatic cholangiocarcinoma: different characters of perihilar large duct type versus peripheral small duct type. J Hepatobiliary Pancreat Sci 22: 94-100, 2015.

10 Asayama Y, Yoshimitsu K, Irie H, Tajima T, Nishie A, Hirakawa M, Nakayama T, Kakihara D, Taketomi A, Aishima S and Honda $\mathrm{H}$ : Delayed-phase dynamic CT enhancement as a prognostic factor for mass-forming intrahepatic cholangiocarcinoma. Radiology 238: 150-155, 2006.

11 Altaee MY, Johnson PJ, Farrant JM and Williams R: Etiologic and clinical characteristics of peripheral and hilar cholangiocarcinoma. Cancer 68: 2051-2055, 1991.

12 Madariaga JR, Iwatsuki S, Todo S, Lee RG, Irish W and Starzl TE: Liver resection for hilar and peripheral cholangiocarcinomas: a study of 62 cases. Ann Surg 227: 70-79, 1998.

13 Nakajima T, Kondo Y, Miyazaki $M$ and Okui K: A histopathologic study of 102 cases of intrahepatic cholangiocarcinoma: histologic classification and modes of spreading. Hum Pathol 19: 1228-1234, 1988.

14 Okuda K, Kubo Y, Okazaki N, Arishima T and Hashimoto M: Clinical aspects of intrahepatic bile duct carcinoma including hilar carcinoma: a study of 57 autopsy-proven cases. Cancer 39: 232-246, 1966.

15 Robles R, Figueras J, Turrión VS, Margarit C, Moya A, Varo E, Calleja J, Valdivieso A, Valdecasas JC, López P, Gómez M, de Vicente E, Loinaz C, Santoyo J, Fleitas M, Bernardos A, Lladó L, Ramírez P, Bueno FS, Jaurrieta E and Parrilla P: Spanish experience in liver transplantation for hilar and peripheral cholangiocarcinoma. Ann Surg 239: 265-271, 2004.

16 Fan B, Malato Y, Calvisi DF, Naqvi S, Razumilava N, Ribback S, Gores GJ, Dombrowski F, Evert M, Chen X and Willenbring $\mathrm{H}$ : Cholangiocarcinomas can originate from hepatocytes in mice. J Clin Invest 122: 2911-2915, 2012.

17 Sekiya S and Suzuki A: Intrahepatic cholangiocarcinoma can arise from Notch-mediated conversion of hepatocytes. J Clin Invest 122: 3914-3918, 2012.

18 Palmer WC and Patel T: Are common factors involved in the pathogenesis of primary liver cancers? A meta-analysis of risk factors for intrahepatic cholangiocarcinoma. J Hepatol 57: 6976, 2012.

19 Li M, Li J, Li P, Li H, Su T, Zhu R and Gong J: Hepatitis B virus infection increases the risk of cholangiocarcinoma: a metaanalysis and systematic review. J Gastroenterol Hepatol 27: 1561-1568, 2012.

20 Lee TY, Lee SS, Jung SW, Jeon SH, Yun SC, Oh HC, Kwon S, Lee SK, Seo DW, Kim MH and Suh DJ: Hepatitis B virus infection and intrahepatic cholangiocarcinoma in Korea: a casecontrol study. Am J Gastroenterol 103: 1716-1720, 2008.

21 Sulpice L, Rayar M, Boucher E, Pracht M, Meunier B and Boudjema K: Treatment of recurrent intrahepatic cholangiocarcinoma. Br J Surg 99: 1711-1717, 2012.

22 Souche R, Addeo P, Oussoultzoglou E, Herrero A, Rosso E, Navarro F, Fabre JM and Bachellier P: First and repeat liver resection for primary and recurrent intrahepatic cholangiocarcinoma. Am J Surg 212: 221-229, 2016.

23 Sur MD, In H, Sharpe SM, Baker MS, Weichselbaum RR, Talamonti MS and Posner MC: Defining the Benefit of Adjuvant Therapy Following Resection for Intrahepatic Cholangiocarcinoma. Ann Surg Oncol 22: 2209-2217, 2015.

24 Mavros MN, Economopoulos KP, Alexiou VG and Pawlik TM: Treatment and Prognosis for Patients With Intrahepatic Cholangiocarcinoma: Systematic Review and Meta-analysis. JAMA Surg 149: 565-574, 2014.

25 Ray CE Jr, Edwards A, Smith MT, Leong S, Kondo K, Gipson M, Rochon PJ, Gupta R, Messersmith W, Purcell T and Durham $\mathrm{J}$ : Metaanalysis of survival, complications, and imaging response following chemotherapy-based transarterial therapy in patients with unresectable intrahepatic cholangiocarcinoma. J Vasc Interv Radiol 24: 1218-1226, 2013.

26 Shimoda M, Farmer DG, Colquhoun SD, Rosove M, Ghobrial RM, Yersiz H, Chen P and Busuttil RW: Liver transplantation for cholangiocellular carcinoma: analysis of a single-center experience and review of the literature. Liver Transpl 7: 10231033, 2001.

27 Pascher A, Jonas S and Neuhaus P: Intrahepatic cholangiocarcinoma: indication for transplantation. J Hepatobiliary Pancreat Surg 10: 282-287, 2003.

$28 \mathrm{Fu} \mathrm{Y}$, Yang W, Wu W, Yan K, Xing BC and Chen $\mathrm{MH}$ : Radiofrequency ablation in the management of unresectable intrahepatic cholangiocarcinoma. J Vasc Interv Radiol 23: 642$649,2012$.
Received October 9, 2016

Revised October 25, 2016

Accepted November 1, 2016 